\title{
Mycobiota and Fungal Metabolites in Improved Groundnut Varieties in Nigeria
}

\author{
Toba S. Anjorin ${ }^{1, *}$, Alhassan Usman ${ }^{2}$, Stephen O. Fapohunda ${ }^{3}$, Adobe Kwanashie ${ }^{4}$, Michael \\ Sulyok ${ }^{5}$ and Rudolf Krska ${ }^{5}$ \\ ${ }^{1}$ Department of Crop Protection, Faculty of Agriculture, University of Abuja, PMB 117, Abuja, Nigeria; ${ }^{2}$ Department of Plant Science, \\ Institute of Agriculture Research, Faculty of Agriculture, Ahmadu Bello University, Zaria, Nigeria; ${ }^{3}$ Department of Microbiology, Babcock \\ University, Ilishan ;remo, Ogun State, Nigeria; ${ }^{4}$ Department of Crop Protection, Ahmadu Bello University, Zaria, Nigeria; ${ }^{5}$ Center for \\ Analytical Chemistry, Department of Agrobiotechnology (IFA-Tulln), University of Natural Resources and Life Sciences Vienna (BOKU), \\ Konrad Lorenzstr, 20, A-3430 Tulln, Austria
}

Received: April 4, 2020; Revised: Sept 18, 2020; Accepted: Nov 10, 2020

\begin{abstract}
The study investigated the incidence of mycobiota, Aspergillus spp metabolites and aflatoxin biosynthesis precursors in 60 samples of seeds and haulms of improved groundnut genotypes in Nigeria. Culturing of the infected seeds was done using the agar plate method and mycobiota identification was done using conventional cultural and microscopy method. The occurrence of Aspergillus toxins, and aflatoxin biosynthesis precursors were evaluated using liquid chromatography-mass spectrometry (LC- MS/MS). Three identified species and four unidentified species of fungi were isolated from the seeds with Aspergillus niger (47.19\%) and A. flavus (17.82\%) having higher incidence more than others. The seed samples from ICGX 86024 and ICGX 01276 had the highest and lowest fungal incidence respectively. No aflatoxin $\mathrm{B}_{1}$, ochratoxin A or fumonisins were detected in the seeds and haulms samples of the improved groundnut despite the incidence of $A$. flavus. Fifteen fungal metabolites were found including eight Aspergillus metabolites and seven aflatoxin biosynthesis precursors. From our result, STC which is involved in flatoxin biosynthesis pathway was below limit of detection and thus aflatoxin formation in the groundnuts might be interrupted. This report is foremost in elucidating the aflatoxins biosynthesis precursors in seeds and haulms of improved groundnut varieties in Northern Nigeria.
\end{abstract}

Keywords: Aflatoxin precursors, Aspergillus metabolites, groundnut, haulm, seeds, incidence, mycobiota.

\section{Introduction}

Groundnut (Arachis hypogaea L.) is a valuable oilseed and food legume crop in the savannah and semi-arid regions of the world. It is a commercial crop for local markets as well as for export in many developed and developing countries (Guchi, 2015a). Major groundnut producing areas in Nigeria are in the Central, Eastern and Western Northern zones (Ntare, 2007; Ifeji et al., 2014; Vabi et al., 2018). The production of quality marketable seeds especially from local varieties and some improved ones are constrained by Aspergillus species (Guchi, 2015b). The fungus often causes quantitative losses and produces highly poisonous chemical substances called aflatoxins. Aflatoxins are produced by Aspergillus species which are soil-borne fungi that occur Worldwide (Granados-Chinchilla, 2017; Gruber-Dorninger et al, 2017).

In an attempt to improve groundnut $\mathrm{v}$

arieties for resistance against fungal foliar diseases and aflatoxins, breeding techniques and recombinant DNA technology are now employed (Dwivedi 2003; BhatnagarMathur, 2015). The International Crops Research Institute for the Semi-Arid Tropics (ICRISAT) in West Africa has bred and released groundnut varieties that are high yielding in terms of the pods and fodders and also free of aflatoxins.

Though some improved groundnut cultivars were bred to resist aflatogenic fungi and aflatoxins formation by the crop breeders, there are several other minor mycotoxins or metabolites that could be produced in their seeds or in the haulms. There is however paucity of multi-mycotoxin research-based on such roughages within animal feed chain in developing countries like Nigeria. This investigation was therefore carried out to determine the incidence of mycobiota, the profile of Aspergillus metabolites and aflatoxin biosynthesis precursors in the seeds and haulms of improved groundnut varieties in Nigeria using multi-mycotoxins LC-MS/MS. The study has risk assessment significance and also gives plausible explanation to how improved groundnut varieties with the presence of Aspergillus flavus resist aflatoxin biosynthesis.

\section{Materials and Methods}

\subsection{Experimental field}

The experimental groundnut varieties were grown on farmers` participatory lines trial at location T19, Institute of Agricultural Research (IAR) Experimental Field,

\footnotetext{
* Corresponding author e-mail: toba.anjorin@uniabuja.edu.ng.
} 
Ahmadu Bello University, Samaru Zaria, Kaduna State, Nigeria (Long. $7^{\circ} 38^{\prime}$ and $7^{\circ} 50^{\prime} \mathrm{E}$ and Lat.11 ${ }^{\circ} 01^{\prime}$ and 11 $10^{\prime} \mathrm{N}$ ) with altitude of $686 \mathrm{~m}$ above sea level under rain-fed conditions in the Northern Guinea Savannah of Nigeria. The mean daily air temperature of the area during the planting season was $24.3^{\circ} \mathrm{C}$, while the mean annual rainfall was $1201 \mathrm{~mm}$. The soil texture of the field was sandy loam and with a mean soil $\mathrm{pH}$ of 5.5. The unit plot size was $5 \mathrm{~m} \times 3 \mathrm{~m}$, and they were laid out in a randomized complete block design (RCBD) with each treatment replicated thrice. The plant spacing was $30 \mathrm{~cm}$ and $15 \mathrm{~cm}$ while plot to plot and replication to replication distance was 0.5 and $1.0 \mathrm{~m}$, respectively. Total area of the farm was $17 \mathrm{~m} \times 37 \mathrm{~m}$ i.e. $629 \mathrm{~m}^{2}$. The seeds of eight groundnut genotype (Short duration - ICGV-IS 07999, ICGV 94379 and ICGV 86024; Medium duration - ICGV 01276, ICGVIS-09992, ICGX 24, ICGV-1S-09926 and ICGV 08540) were collected from International Crops Research Institute for the Semi-Arid Tropics (ICRISAT). Also seeds of two local checks - medium-maturing SAMNUT 22 and earlymaturing SAMNUT 24 collected from IAR\&T were all sown on the flat at the mean depth of $3.5 \mathrm{~cm}$ on the 3rd week of July, 2018. Each of the plots was applied with basal dose of 20:40:20 NPK fertilizer at $400 \mathrm{~kg} \mathrm{ha}^{-1}$.

\subsection{Sample collection}

The haulms of each groundnut variety from each plot were separately collected and properly packed in polystyrene bags and then labelled accordingly. Harvested dried unshelled samples were collected in brown envelopes from the farm store in IAR, Samaru, before being transferred to and kept in the Mycotoxin and Pesticidal Residue laboratory of Department of Crop Protection, ABU, Zaria. Prior to commencing laboratory analysis procedures on the samples which was within a month of harvest, they were stored at $4^{\circ} \mathrm{C}$ to prevent further fungal growth and metabolite production within the samples (Garcia-Cela et al., 2020). Each sample was divided into two portions. The samples from one portion were used for mycological analysis. The second portion was separately packed in zip lock and transported in to University of Natural Resources and Life Sciences Vienna (BOKU), Tulln, Austria for multi-mycotoxins analysis.

Identification of fungal species using Agar plate method. The Potato Dextrose Agar (PDA) was prepared following the method of Giorni et al., 2019. It was then autoclaved, allowed to cool and poured aseptically in the Petri dishes. When the PDA was about to gel, four seeds from each groundnut genotypes were aseptically plated in the Petri dishes. The experimental design was complete randomized design and replicated 4 times. The individual colony of fungi species growing on the agar was examined after seven days of plating. The identification of fungi was carried out based on the colony characters and morphology of the fungus under stereoscopic microscope and by referring to the production of food-borne fungi manual (Joanne et al., 2008).

\subsection{Analysis of mycotoxin}

\subsubsection{Reagents}

Liquid chromatographic grade of methanol $\left(\mathrm{CH}_{3} \mathrm{COOH}\right)$ and acetonitrile were purchased from Merck (Germany) and VWR (Belgium) respectively. The Mass Spectrometry grade ammonium acetate and standards for fungi metabolite were brought from Sigma-Aldrich (Austria). Decontamination of water was carried out consecutively through reverse osmotic pressure and ultra analytic system was purchased from Veolia water (UK). A total of 34 working solutions were made and kept at $-20{ }^{\circ} \mathrm{C}$ in the refrigerator (Labcold $\AA$, FKvsl 4113, Fishers Scientific, UK) but were brought to $25{ }^{\circ} \mathrm{C}$ before use. Fresh final working solution was mixed accordingly for the spiking experiment.

\subsection{Groundnut Extraction}

Each grain and haulm samples were milled using a cyclone pulverizer which has one millimetre square sieve (Cyclotech, Sweden) before being homogenized. Five grams each were measured into the centrifuge tube $(0.05 \mathrm{~L}$ polypropylene). Twenty millilitres of the separation solvent (acetic acid/water/acetonitrile 1:20:79, v/v/v) were added before being vortexed using laboratory rotary shaker (Model GFL 3017, Germany). Ratio of the dilution of the sample with the solvent was 1:1 and 5 millilitres of the dilution obtained from the extract was shot into the LCMS/MS.

\subsection{LC-MS/MS Parameters}

Analysis of the extracts was achieved with a QTrap 5500 multimycotoxin LC-MS/MS system (Applied Biosystem, California, United State of America) furnished with Turbolon spray ESI source and High Performance Liquid Chromatography (Agilent, Germany). Other liquid chromatography/Mass Spectrometry protocols applied for chromatographic separation, identifying analytes that are positive, quantification of liquid standard identification were as described by Malachova et al., 2015. The method precision was tested through proficiency testing organized by Bureau Interprofessionel des Etudes Analytique (BIPEA) (Gennevilliers, France) in accordance with ISO $13525: 2015$. All the 60 results of the groundnut were between $-2<\mathrm{x}<2$ which was a satisfactory range.

Maximum and median concentration $\left(\mu \mathrm{gkg}^{-1}\right)$ of toxins and metabolites were determined from the data collected for each of the samples analysed. The fungi isolated from the seeds of the improved groundnut varieties were tabulated based on their species. The mean percentage of seeds infected with fungi per treatment were separated with Tukey's test at 5\% level of probability.

\section{Results}

\subsection{Mycobiota load in the groundnut seeds}

The three Aspergillus species detected in the groundnut seed samples were Aspergillus niger, A. flavus and $A$. fumigatus (Table 1, Figures 1 and 2). Other four genera observed were Penicillium spp, Rhizopus spp, Fusarium spp and Sclerotium spp as shown in Table 1. The percentage of SAMNUT 22 (100\%), SAMNUT 24 (96.67\%), ICGV-1S-09992 (96.67\%) and ICGV 94379 $(100 \%)$ seeds infected with fungi were significantly higher $(p \leq 0.05)$ than other varieties. There were total of 303 fungal isolates recovered from all samples, out of which 143 (47.19\%) and 54 (17.82\%) were A. niger and A. flavus respectively. The seed samples from ICGX 86024 and ICGX 01276 had the highest and lowest total fungal incidence respectively. 
Table 1. Incidence of fungi isolated from culture plate of seeds of improved groundnut varieties at 5 Days After Inoculation *Means with the same alphabet(s) within the column are not significantly different from one another using Tukey's test at $5 \%$ probability level.

\begin{tabular}{|c|c|c|c|c|c|c|c|c|c|c|}
\hline \multirow[t]{2}{*}{ S/No } & \multirow{2}{*}{$\begin{array}{l}\text { Variety } \\
\text { /genotype }\end{array}$} & \multirow{2}{*}{$\begin{array}{l}\text { \% of } \\
\text { infected } \\
\text { seeds }\end{array}$} & \multicolumn{8}{|c|}{ Incidence of fungal genera and species } \\
\hline & & & $\begin{array}{l}\text { Aspergillus } \\
\text { niger }\end{array}$ & $\begin{array}{l}\text { A. } \\
\text { flavus }\end{array}$ & $\begin{array}{l}\text { A. } \\
\text { fumigatus }\end{array}$ & $\begin{array}{l}\text { Fusarium } \\
\text { sp. }\end{array}$ & Rhizopus sp. & $\begin{array}{l}\text { Sclerotium } \\
\text { sp. }\end{array}$ & $\begin{array}{l}\text { Penicillium } \\
\text { sp. }\end{array}$ & $\begin{array}{l}\text { Total fungi } \\
\text { incidence }\end{array}$ \\
\hline 1 & SAMNUT 22 & $100^{e} *$ & 18 & 10 & 0 & 1 & 0 & 0 & 0 & 29 \\
\hline 2 & SAMNUT 24 & $96.67^{\mathrm{e}}$ & 10 & 8 & 0 & 8 & 6 & 6 & 0 & 38 \\
\hline 3 & ICGV-1S-09992 & $96.67^{\mathrm{e}}$ & 20 & 9 & 0 & 1 & 1 & 0 & 0 & 31 \\
\hline 4 & ICGX 24 & $46.67^{\mathrm{a}}$ & 7 & 2 & 0 & 3 & 12 & 0 & 1 & 25 \\
\hline 5 & ICGX 86024 & $100^{\mathrm{e}}$ & 19 & 8 & 0 & 7 & 0 & 2 & 0 & 36 \\
\hline 6 & ICGV -1S-09926 & $93.33^{d}$ & 10 & 5 & 0 & 0 & 0 & 17 & 0 & 32 \\
\hline 7 & ICGV 01276 & $83.33^{b}$ & 12 & 0 & 0 & 7 & 0 & 0 & 0 & 19 \\
\hline 8 & ICGV 07999 & $90.00^{\mathrm{c}}$ & 3 & 1 & 0 & 0 & 4 & 22 & 0 & 30 \\
\hline 9 & ICGV SM-08540 & $93.33^{\mathrm{d}}$ & 23 & 4 & 1 & 0 & 2 & 2 & 0 & 32 \\
\hline \multirow[t]{3}{*}{10} & ICGV 94379 & $100^{\mathrm{e}}$ & 21 & 7 & 0 & 2 & 0 & 0 & 0 & 30 \\
\hline & Total (\%) & & 143 & 54 & 1 & 29 & 25 & 49 & 1 & 302 \\
\hline & & & $(47.2)$ & $(17.8)$ & $(0.003)$ & $(9.6)$ & (8.3) & $(16.2)$ & $(0.003)$ & (100) \\
\hline
\end{tabular}

Macroscopic observation of $A$. fumigatus showed blue grayish coloured ((Figure1, A1) and orange coloured in reverse also with powdery texture and wrinkle in reverse (B1).The colony morphology of A. niger observed reveals a black colour colony on the top (A2) and sulphur-yellow colonies on the reverse (B2). Downy and powdery textured and grow fast within 2 to 4 days. A. flavus colony was

A. fumigatus

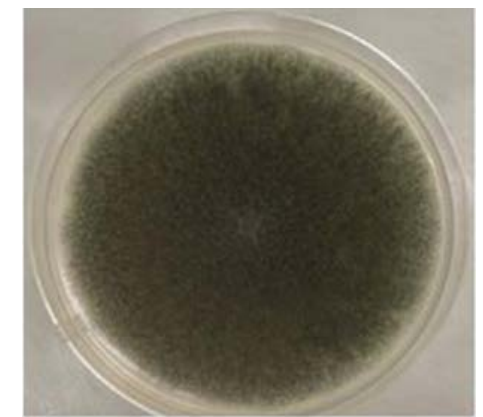

A. fumigatus

A1

A. niger
A. niger

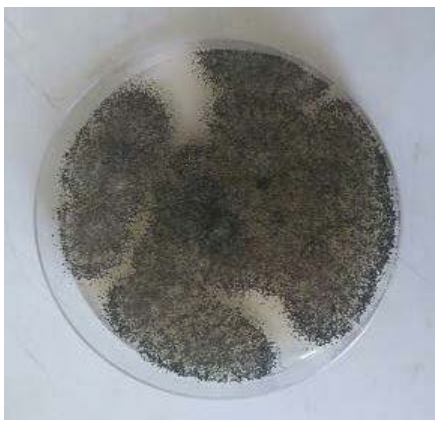

A2

A. flavus

Plate A. Colony features (Front surface)

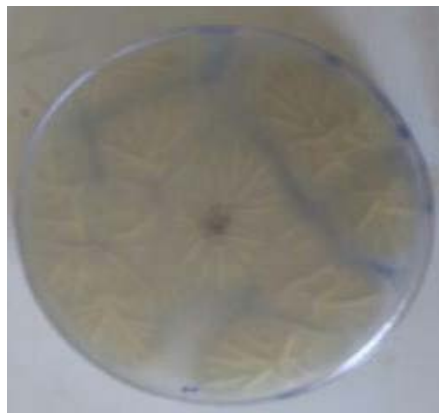

B2 white and yellowish green on the surface and powdery on the surface of the colony (A3); it was also pale yellow with downy texture on the base on reverse (B3). The mycelia grew filled the plate within 5 to 6 days.

The microscopic features of the three identified Aspergillus species is as shown in Figures 2 and Table 2.

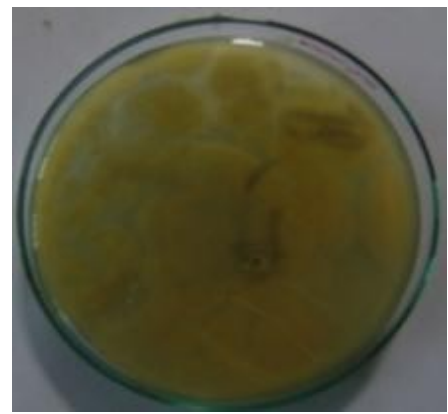

B1

Plate B. Reverse surface

A. flavus
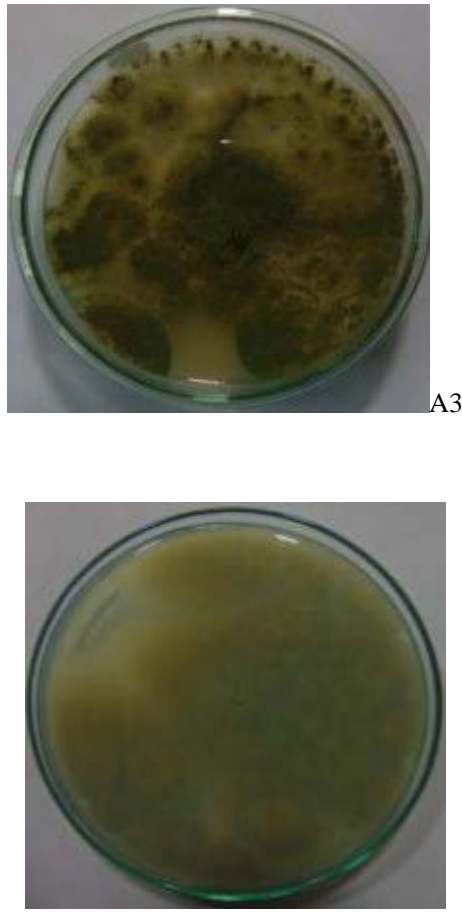

B3 


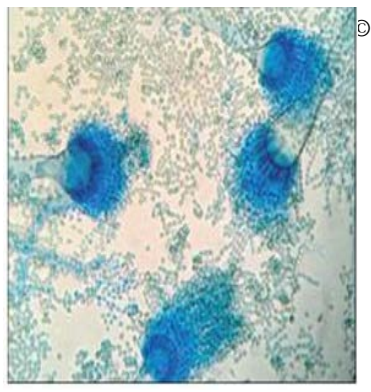

C1.

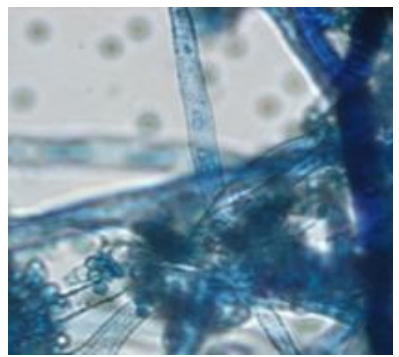

D1

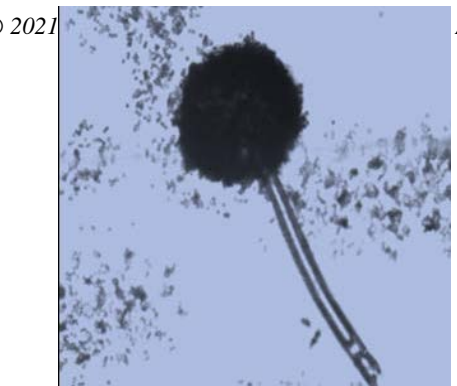

C2

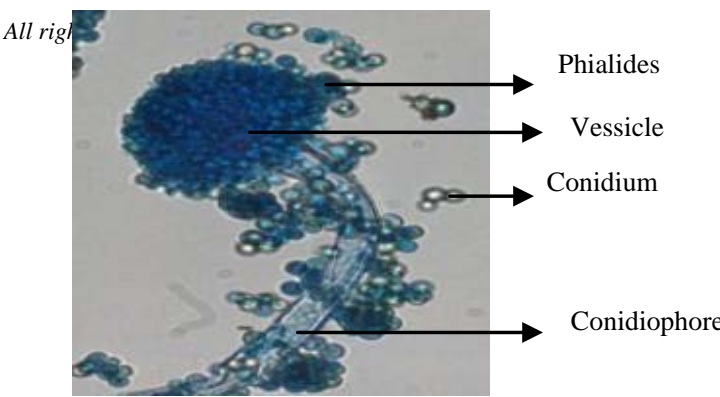

C3

Conidial heads (frutiting bodies)

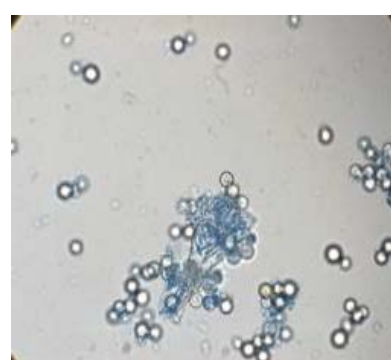

D2

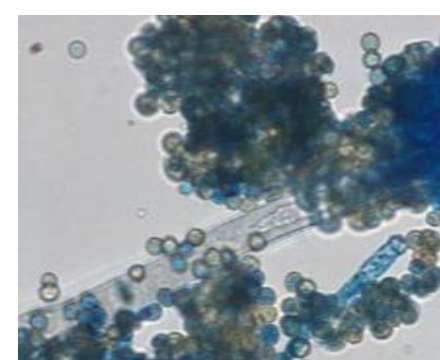

D3

Conidia (x 400)

Figure 2. Microscopic features of the three identified Aspergillus species. Conidial heads (C1-C3) and conidia - x400 (D1-D3)

Table 2. Microscopic characteristics of the identified Aspergillus spp isolates

\begin{tabular}{|c|c|c|c|c|c|c|c|c|}
\hline Fungus & Microscop & ic features & & & & & & \\
\hline $\begin{array}{l}\text { Aspergillus } \\
\text { species }\end{array}$ & $\begin{array}{l}\text { Size } \\
(\mu \mathrm{m})\end{array}$ & Stipes colour & Surface & $\begin{array}{l}\text { Vesicle } \\
\text { serration }\end{array}$ & Vessicle size & $\begin{array}{l}\text { Metula } \\
\text { covering }\end{array}$ & Shape & Conidia surface \\
\hline A. flavus & $400-800$ & $\begin{array}{l}\text { Pale brown } \\
\text { roughened }\end{array}$ & $\begin{array}{l}\text { Quietly } \\
\text { spherical }\end{array}$ & Biserate & $30-40 \mu \mathrm{m})$ & $2 / 3$ & Glubose ellipsoid & $\begin{array}{l}\text { Smooth finely } \\
\text { roughened }\end{array}$ \\
\hline A. niger & $400-3000$ & Slighlty brown & $\begin{array}{l}\text { Smooth } \\
\text { walled }\end{array}$ & $\begin{array}{l}\text { Biserate, large } \\
\text { size }\end{array}$ & $15.45-20.45 \mu \mathrm{m}$ & entirely & Glubose & $\begin{array}{l}\text { Very rough, } \\
\text { irregular }\end{array}$ \\
\hline A. fumigatus & $200-400$ & Grayish near ape & $\begin{array}{l}\text { Smooth } \\
\text { walled }\end{array}$ & $\begin{array}{l}\text { Uniserate, } \\
\text { pyriform }\end{array}$ & $15.45-20.45 \mu \mathrm{m}$ & Upper2/3 & $\begin{array}{l}\text { Globose, small in } \\
\text { columns }\end{array}$ & $\begin{array}{l}\text { Smooth or } \\
\text { spinose }\end{array}$ \\
\hline
\end{tabular}

\subsection{Occurrence of fungal toxins and metabolites in} groundnut seeds and haulms

Major mycotoxins such as aflatoxin $\mathrm{B}_{1}$, ochratoxin $\mathrm{A}$, fumonisin $B_{1}$, fumonisin $B_{2}$, fumonisin $B_{3}$, fumonisin $B_{4}$, fumonisin $B_{6}$ were below the limit of detection by LCMS/MS analyser in all the seeds and haulms of the ten groundnut genotypes investigated. The occurrence of 8 emerging Aspergillus toxins, sterigmatocystin (STC) and other six biosynthesis precursors were detected in the groundnut and haulm samples. In addition, a bacterial toxin; monactin $\left(0.017 \mu \mathrm{g} \mathrm{kg}^{-1}\right)$ was detected in the haulm of variety ICGV-1509926 (Tables 3 and 4). The only bacterial toxin detected was monactin found in the haulm of variety ICGV-1509926 with a low median concentration of $0.017 \mu \mathrm{g} \mathrm{kg}^{-1}$.

No Aspergillus toxin and metabolites were detected in the seeds of ICGV-IS-09992 and ICGV-IS-09926. Also, no toxins and metabolites were detected in the haulms of ICGV-IS-09992. The seeds of ICGV-85024 had the highest co-occurrence of seven out of eight Aspergillus emerging toxins and metabolites, while in the haulms, the highest co-occurrence of six metabolites was found in ICGV-1S-09926. Lower occurrence and concentration of Aspergillus toxins and metabolites were detected in the seeds of ICGV-01276, ICGV-SM 08540, SAMNUT 22 and SAMNUT 24 varieties than in their haulms (Table 3).

High median concentration of 3-Nitropriopinic acid (2716.3 $\mu \mathrm{g} \mathrm{kg}^{-1}$ ) was detected in the in seeds of ICGV94379. Also, highest median concentration of 3Nitropropanoic acid (3716 $\mu \mathrm{g} \mathrm{kg}^{-1}$ ) was detected in the haulms of the ICGV-94379 genotype.

Only the seeds of ICGV- IS-09926 had all the seven aflatoxin biosynthesis precursors including STC (Table 4). The median concentration level of STC in the seeds of ICGV-1S-09926 had a maximum concentration of $2.6 \mu \mathrm{g}$ kg- ${ }^{1}$ with a median of $0.87 \mathrm{\mu gkg-}^{1}$. The result also revealed that that 7 out of the 10 groundnut varieties' seeds were contaminated with versicolorin A, versicolorin C, and averufin (Table 3), but none of the aflatoxin biosynthesis precursors was detected in the haulms of ICGV- 07999, ICGV-24, ICGV-1S-09926, ICGV-85024. 
Table 3. Maximum and median concentration $\left(\mu \mathrm{g} \mathrm{kg}^{-1}\right) *$ of Aspergillus metabolites in groundnut seeds and haulms

\begin{tabular}{|c|c|c|c|c|c|c|c|c|c|c|c|c|c|c|c|c|c|c|c|}
\hline \multirow{2}{*}{$\begin{array}{l}\text { Groundnut } \\
\text { genotype/ } \\
\text { Aspergillus } \\
\text { metabolite }\end{array}$} & \multicolumn{2}{|c|}{$\begin{array}{l}\text { ICGV- } \\
07999\end{array}$} & \multicolumn{2}{|c|}{$\begin{array}{l}\text { ICGV- } \\
24\end{array}$} & \multicolumn{2}{|c|}{$\begin{array}{l}\text { ICGV- } \\
01276\end{array}$} & $\begin{array}{l}\text { ICGV-1S- } \\
09926\end{array}$ & \multicolumn{2}{|c|}{$\begin{array}{l}\text { ICGV- } \\
1 \mathrm{~S} \\
09992 \\
\end{array}$} & \multicolumn{2}{|c|}{ ICGV-85024 } & \multicolumn{2}{|l|}{$\begin{array}{l}\text { ICGV- } \\
94379\end{array}$} & \multicolumn{2}{|c|}{$\begin{array}{l}\text { ICGV-SM } \\
08540\end{array}$} & \multicolumn{2}{|c|}{$\begin{array}{l}\text { SAMNUT } \\
22\end{array}$} & \multicolumn{2}{|c|}{$\begin{array}{l}\text { SAMNUT } \\
24\end{array}$} \\
\hline & Sd & Hlm & Sd & Hlm & Sd & $\mathrm{Hlm}$ & Sd Hlm & Sd & Hlm & Sd & Hlm & Sd & $\mathrm{Hlm}$ & Sd & $\mathrm{Hlm}$ & Sd & Hlm & Sd & Hlm \\
\hline $\begin{array}{l}\text { 3-Nitropropionic } \\
\text { acid }\end{array}$ & $\begin{array}{l}0.4 \\
(0.1)\end{array}$ & $\begin{array}{l}1.0 \\
(0.6)\end{array}$ & $\begin{array}{l}0.3 \\
(0.1)\end{array}$ & - & - & - & $-\quad-$ & - & - & $\begin{array}{l}0.3 \\
(0.1)\end{array}$ & - & $\begin{array}{l}8149 \\
(2716)\end{array}$ & $\begin{array}{l}8279 \\
(3716)\end{array}$ & - & $\begin{array}{l}0.4 \\
(0.14)\end{array}$ & - & - & - & - \\
\hline gragillin & & - & - & - & - & & $\begin{array}{ll}5533 \\
-\quad(1844)\end{array}$ & - & - & - & - & - & - & - & $\begin{array}{l}7279 \\
(2426)\end{array}$ & - & - & - & - \\
\hline Malformin A & - & - & - & - & - & $\begin{array}{l}0.04 \\
(0.01)\end{array}$ & $\begin{array}{l}0.07 \\
(0.02)\end{array}$ & - & - & $\begin{array}{l}0.01 \\
(0.003)\end{array}$ & - & - & $\begin{array}{l}5.7 \\
(1.8)\end{array}$ & - & - & - & - & - & - \\
\hline Malformin C & - & - & - & - & - & - & $\begin{array}{ll}0.02 \\
-\quad(0.02)\end{array}$ & - & - & $\begin{array}{l}0.03 \\
(0.01)\end{array}$ & - & - & $\begin{array}{l}0.4 \\
(0.14)\end{array}$ & - & - & - & - & - & - \\
\hline Kojic acid(KJ) & - & - & $\begin{array}{l}5.2 \\
(1.7)\end{array}$ & $\begin{array}{l}7.5 \\
(3.2)\end{array}$ & - & - & $\begin{array}{l}4.6 \\
-\quad(1.5)\end{array}$ & - & - & $\begin{array}{l}6.7 \\
(2.2)\end{array}$ & - & $\begin{array}{l}8149 \\
(2716)\end{array}$ & $\begin{array}{l}1889 \\
(630)\end{array}$ & - & $\begin{array}{l}6.6 \\
(2.2)\end{array}$ & - & - & - & $\begin{array}{l}6.1 \\
(2)\end{array}$ \\
\hline Methyl funicone & - & - & $\begin{array}{l}0.7 \\
(0.2)\end{array}$ & $\begin{array}{l}0.1 \\
(0.01)\end{array}$ & - & $\begin{array}{l}0.2 \\
(0.07)\end{array}$ & $\begin{array}{ll} & 0.8 \\
- & (0.3)\end{array}$ & - & - & $\begin{array}{l}0.3 \\
(0.1)\end{array}$ & - & - & . & - & $\begin{array}{l}1.16 \\
(0.4)\end{array}$ & - & $\begin{array}{l}0.04 \\
(0.01)\end{array}$ & - & $\begin{array}{l}0.5 \\
(0.2)\end{array}$ \\
\hline Emericellamide A & - & - & - & - & $\begin{array}{l}1516 \\
(505)\end{array}$ & $\begin{array}{l}1670 \\
(605)\end{array}$ & $\begin{array}{r}1156 \\
-\quad(385)\end{array}$ & - & - & $\begin{array}{l}2272 \\
(757)\end{array}$ & - & $\begin{array}{l}2.03 \\
(0.7)\end{array}$ & $\begin{array}{l}4.6 \\
(0.2)\end{array}$ & $\begin{array}{l}2748 \\
(978)\end{array}$ & $\begin{array}{l}2948 \\
(998)\end{array}$ & $\begin{array}{l}2663 \\
(888)\end{array}$ & $\begin{array}{l}2863 \\
(890)\end{array}$ & $\begin{array}{l}5642 \\
(1881 \\
)\end{array}$ & $\begin{array}{l}6642 \\
(2881 \\
)\end{array}$ \\
\hline Sydowinin A & & - & - & $\begin{array}{l}1.21 \\
(0.3)\end{array}$ & - & - & - & - & - & $\begin{array}{l}1.13 \\
(0.4)\end{array}$ & - & - & - & - & - & - & - & - & - \\
\hline
\end{tabular}

- = < limits of detection; sd = seed; hlm= haulm

Table 4. Maximum and median concentration $\left(\mu \mathrm{g} \mathrm{kg}^{-1}\right)$ of sterigmatocystin (STC) and other aflatoxin biosynthesis precursors in groundnut seeds and haulms

\begin{tabular}{|c|c|c|c|c|c|c|c|c|c|c|c|c|c|c|c|c|c|c|c|c|}
\hline \multirow[t]{2}{*}{ G/nut genotype } & \multicolumn{2}{|c|}{$\begin{array}{l}\text { ICGV- } \\
07999\end{array}$} & \multicolumn{2}{|c|}{ ICGV-24 } & \multicolumn{2}{|c|}{$\begin{array}{l}\text { ICGV- } \\
01276\end{array}$} & \multicolumn{2}{|c|}{$\begin{array}{l}\text { ICGV-1S- } \\
09926 \\
\end{array}$} & \multicolumn{2}{|c|}{$\begin{array}{l}\text { ICGV-1S- } \\
09992\end{array}$} & \multicolumn{2}{|c|}{ ICGV-85024 } & \multicolumn{2}{|c|}{ ICGV-94379 } & \multicolumn{2}{|c|}{$\begin{array}{l}\text { ICGV-SM } \\
08540\end{array}$} & \multicolumn{2}{|c|}{ SAMNUT22 } & \multicolumn{2}{|c|}{ SAMNUT24 } \\
\hline & Sd & Hlm & Sd & Hlm & Sd & Hlm & $\mathrm{Sd}$ & Hlm & Sd & Hlm & Sd & Hlm & Sd & Hlm & Sd & Hlm & Sd & Hlm & Sd & Hlm \\
\hline Sterigmatocystin & - & - & - & - & - & - & $\begin{array}{l}2.6 \\
(0.87)\end{array}$ & - & - & - & - & - & - & - & - & - & - & - & - & - \\
\hline Nidurufin & $\begin{array}{l}6.4 \\
(2.1)\end{array}$ & - & $\begin{array}{l}21.9 \\
(1.0)\end{array}$ & - & $\begin{array}{l}2.1 \\
(0.8)\end{array}$ & $\begin{array}{l}1.13 \\
(0.38\end{array}$ & $\begin{array}{l}23.88 \\
(7.96)\end{array}$ & - & $\begin{array}{l}0.03 \\
(0.1)\end{array}$ & $\begin{array}{l}14.14 \\
(4.7)\end{array}$ & $\begin{array}{l}6.8 \\
(2.3)\end{array}$ & - & $\begin{array}{l}0.04 \\
(0.01)\end{array}$ & $\begin{array}{l}68.3 \\
(22.8)\end{array}$ & - & $\begin{array}{l}17.38 \\
(5.75)\end{array}$ & - & $\begin{array}{l}3.33 \\
(1.1)\end{array}$ & - & $\begin{array}{l}47.15 \\
(16)\end{array}$ \\
\hline Versicolorin A & $\begin{array}{l}9.6 \\
(3.2)\end{array}$ & - & $\begin{array}{l}37.8 \\
(13)\end{array}$ & - & $\begin{array}{l}2.3 \\
(0.8)\end{array}$ & $\begin{array}{l}1.26 \\
(0.4)\end{array}$ & $\begin{array}{l}30.55 \\
(10.2)\end{array}$ & - & $\begin{array}{l}0.10 \\
(0.33)\end{array}$ & $\begin{array}{l}16.0 \\
(5.30)\end{array}$ & $8.5(2.8)$ & - & $\begin{array}{l}4.4 \\
(1.7)\end{array}$ & $74.6(25)$ & - & $\begin{array}{l}15.1 \\
(5.02)\end{array}$ & - & $3.6(1.2)$ & - & $\begin{array}{l}48.3 \\
(16.1)\end{array}$ \\
\hline Versicolorin C & $\begin{array}{l}26.1 \\
(8.7)\end{array}$ & - & $\begin{array}{l}91.8 \\
(30.6)\end{array}$ & - & $\begin{array}{l}4.8 \\
(1.5)\end{array}$ & $\begin{array}{l}3.7 \\
(1.23)\end{array}$ & $\begin{array}{l}84.3 \\
(28.1)\end{array}$ & - & $\begin{array}{l}0.19 \\
(0.1)\end{array}$ & $\begin{array}{l}51.99 \\
(13.97)\end{array}$ & $\begin{array}{l}25.4 \\
(8.5)\end{array}$ & - & $\begin{array}{l}5.4 \\
(2.1)\end{array}$ & $213(70.9)$ & - & $\begin{array}{l}56.49 \\
(18.9)\end{array}$ & - & $\begin{array}{l}10.1 \\
(3.4)\end{array}$ & - & $\begin{array}{l}160 \\
(53.3)\end{array}$ \\
\hline Averantin & $\begin{array}{l}0.4 \\
(0.15)\end{array}$ & - & $\begin{array}{l}1.6 \\
(0.5)\end{array}$ & - & $\begin{array}{l}0.9 \\
(0.4)\end{array}$ & $\begin{array}{l}0.07 \\
(0.02\end{array}$ & $\begin{array}{l}1.66 \\
(0.5)\end{array}$ & - &.- & $\begin{array}{l}1.1 \\
(0.4)\end{array}$ & $\begin{array}{l}0.4 \\
(0.13)\end{array}$ & - & - & $3.56(1.2)$ & - & $\begin{array}{l}0.7 \\
(0.2)\end{array}$ & - & $0.19(0.1)$ & - & $2.9(0.9)$ \\
\hline Averufin & $\begin{array}{l}3.4 \\
(1.14)\end{array}$ & - & $\begin{array}{l}13.4 \\
(4.5)\end{array}$ & - & $\begin{array}{l}1.6 \\
(0.9)\end{array}$ & $\begin{array}{l}0.44 \\
(0.13)\end{array}$ & $\begin{array}{l}12.61 \\
(4.2)\end{array}$ & - & $\begin{array}{l}0.08 \\
(0.03)\end{array}$ & $\begin{array}{l}9.11 \\
(3.4)\end{array}$ & $\begin{array}{l}3.29 \\
(1.1)\end{array}$ & - & $\begin{array}{l}0.05 \\
(0.03)\end{array}$ & 25.4 (8.5) & - & $\begin{array}{l}7.3 \\
(2.41)\end{array}$ & - & $\begin{array}{l}1.5 \\
(0.03)\end{array}$ & - & $\begin{array}{l}25.1 \\
(8.4)\end{array}$ \\
\hline $\begin{array}{l}\text { Norsolorinic } \\
\text { acid }\end{array}$ & $\begin{array}{l}0.85 \\
(0.3)\end{array}$ & - & $\begin{array}{l}4.14 \\
(1.4)\end{array}$ & - & - & - & $\begin{array}{l}4.37 \\
(1.4)\end{array}$ & - & - & $\begin{array}{l}2.8 \\
(0.95)\end{array}$ & $\begin{array}{l}1.15 \\
(0.4)\end{array}$ & - & & $10.3(3.8)$ & - & $\begin{array}{l}1.7 \\
(0.57)\end{array}$ & - & $\begin{array}{l}0.5 \\
(0.17)\end{array}$ & - & $8.1(2.7)$ \\
\hline
\end{tabular}

$=<$ limits of detection; sd = seed; hlm= haulm; ( ) = median concentration

\section{Discussion}

Several Aspergillus strains, their metabolites and aflatoxin biosynthesis precursors have been isolated from groundnut seeds and in feeds of ruminants such as cattle feed (Ranjbar et al., 2011) and dairy goat feed from Brazil (da Silva et al., 2015). Ruminant animals are often fed with groundnut haulms as roughages in order to contribute energy and nutrients to their diet (Granados-Chinchilla, 2017). Aspergillus species and aflatoxins have been found to be associated with the silages of corn and sorghum (Alonso et al., 2013). Thus studies concerning safety of animals and humans that consume groundnut and/or their haulms should not be neglected.

Some isolated fungi from Jordan Deseret leaves and fruits were identified as Aspergillus, Alternaria, Rhizopus, Penicillium and Fusarium at the genus level by using macroscopic and microscopic examinations depending on colony colour, shape, hyphae, conidia, conidiophores and arrangement of spores (Alsohaili and Bani-Hasan, 2017) as was carried out in this study. They further molecularly identified the isolated fungi at species level as the extracted fungal DNA was amplified by PCR using specific internal transcribed spacer primer (ITS1/ITS4).

A. niger had the highest incidence (47.20\%) of fungal species isolated from the seeds in this study. It is reported that there are up to145 different secondary metabolites isolated and detected from A. niger (Nielson et al., 2009). These include intricate compounds such as naptho- $\gamma$ pyrones, nigragillin, ochratoxin $\mathrm{A}$ and fumonisin $\mathrm{B}_{2}$. $\mathrm{A}$. flavus with the second highest incidence (17.8\%) is popularly known for being a producer of carcinogenic aflatoxins. About 46 secondary metabolites have been identified in A. flavus, and these non-regulated fungal metabolites include 3-NPA, aflatrem, cyclopionic acid aflavorin, aflavozole, aflavine, aspergillic acid, cladosporin, aspergillic acid, cladosporin and gliotoxin (Adetunji et al., 2014; Cary et al., 2018). Other toxic regulated compound produced by A. flavus is kojic acid 
which is implicated as a neurological poison and mitochondrial toxin (Ahuja, 2008).

The incidence of Sclerotium spp in the samples was 16.2\%. Amber et al. (2012) reported that phenolic acids were detected by HPLC analysis of secondary metabolites in the fungus isolated from chickpea. They observed that this metabolite is responsible for severe collar rot infection in chickpea. The incidence of Fusarium spp in the sampled seeds was 9.6\%. Nesic et al. (2014) identified 10 species of Fusarium and the mycotoxins produced by each of them. F. culmorum is known to be a producer of up to 10 mycotoxins which include fusarins, moniliformin, trichothecenes and zearalenone. Other Fusaria such as $F$. graminearum, F. sporotrichioides, F. crookwellense and $F$. acuminatum can produce up to eight mycotoxins each, while $F$. equiseti and $F$. proliferatum can produce six each, $F$. verticillioides - 4, F. armeniacum -3 and $F$. pseudograminearum - 2 (Berthiller et al. (2013).

The merits of LC-MS/MS based analytical method used in this study include allowing the sensitive concurrent determination of several fungal metabolites in many matrices (Sulyok et al., 2010; Berthiller et al., 2015; Malachova et al., 2018). LC-MS could tremendously assist in the discovery of recently produced mycotoxins (Varga, 2015), as well as hidden (Berthiller, 2013) or other altered forms (Rychlik, 2014) of mycotoxins in crop produce like groundnut.

Major mycotoxins like aflatoxin and their precursors were below level of detection in almost all the groundnut varieties investigated in this study. This is unlike other several previous studies on the natural occurrence of aflatoxins in groundnut samples from other countries. From the samples of stored peanut kernels collected from four different areas in Sudan, Bakhiet et al. (2011) using TLC techniques, reported $\mathrm{AFB}_{1}$ concentration ranges of 17.57 - $404 \mu \mathrm{g} \mathrm{kg}^{-1}$.

Eight Aspergillus metabolites in groundnut seeds and haulms were detected in this study. Nigragillin and Malformin $\mathrm{C}$ are being produced by A. niger (Burdock et al., 2001), Malformin A are produced by A. ficuum, A. awamori and A. phoenicis while Emericellamide A are produced by $A$. nidulans and Sydowinin $A$ by $A$. sydowinin and $A$. versicolor. Kojic acid is known to be produced by A. flavus and A. oryzae, 3- NPA on the other hand is produced by $A$. wentii, A. niger and A. oryzae.

STC was not found in nine out of the 10 improved groundnuts varieties understudied. Out of the 10 analysed improved groundnut varieties, STC was only found in the seeds of ICGV-1S-09926 at low median concentration of $0.87 \mu \mathrm{g} \mathrm{kg}^{-1}$. Sterigmatocystin is normally produced by $A$. flavus, A. nidulans, A. parasiticus and mainly A. versicolor (Versilovskis and De Saeger, 2010). It was reported to often occur where grains and cereal-based food are contaminated with aflatoxigenic fungi (Rank, 2011).

The outcome of this study suggested that the STC (and thus the aflatoxin) pathway is interrupted in the improved groundnut genotypes; thus, such genotypes where not able to produce aflatoxin. Chang et al., (2005) similarly reported that absence of interplay between aflR and aflJ contributes to non-aflatoxigenicity of $A$. sojae. They also reported $a$ sequence stopping points in the aflatoxin biosynthesis gene cluster and flanking zones in non-aflatoxigenic A. flavus isolates. The non-occurrence of aflatoxins in most of the improved groundnut varieties indicated that the use of breeding for resistance could be a better intervention needed in the mitigation of mycotoxin contamination in crops. This is, however, in addition to using cultural practice such as pre-and post-harvest managements, and chemical control methods.

STC is one of the precursors of aflatoxin $B_{1}$ and $G_{1}$ as indicated in a AF biosynthesis pathway as described by Jallow, 2015. Apart from STC being an aflatoxin precursor, it is with toxicity second to $\mathrm{AFB}_{1}$ (Gao et al., 2015). The aflatoxin pathway really requires the presence of STC which often forms Omethylsterigmatocystin (OMST) with the help of Omethyltransferase. OMST could then be converted to $\mathrm{AFB}_{1}$ \& AFG1 with the presence of cytochrome P-450 monooxygenase encoded by ord-1 (Audebert et al., 2014; Bertuzzi et al., 2017; Wartu et al., 2017).

\section{Conclusion}

In this study identification of the fungal isolates up to species was impossible due to non- involvement of molecular characterization techniques. The producing fungus of some of the fungal metabolites detected could not be identified. Thus, molecular identification of fungi in further studies on this subject is necessary in future studies

The analysis indicated that there was no major mycotoxin detected in all the seeds and haulms of the ten groundnut genotypes despite the presence of Aspergillus spp. The improved groundnut varieties might have been bred to resist aflatoxin production, and thus were below detectable level. Planting of such improved varieties with good crop management practices can help reduce aflatoxin and other major mycotoxins contamination in groundnut before storage. In most of the groundnuts analysed, there were incidences of contamination of some minor mycotoxins and metabolites in the seeds more than their haulms.

The non-occurrence of aflatoxins in most of the improved groundnut varieties indicated that the use of breeding for resistance could be a better intervention needed in the mitigation of mycotoxin contamination in crops. This is, however, in addition to using cultural practice such as pre-and post-harvest managements, and chemical control methods. This report is believed to be the first in elucidating the aflatoxins biosynthesis precursors in seeds and haulms of improved groundnut varieties in Northern Nigeria. The result could serve as base information for groundnut breeders to produce a broad spectrum of mycotoxin-resistant groundnut.

\section{Declaration of Interest Statement}

There is no conflict of interests.

\section{References}

Adetunji M, Atanda O, Ezekiel CN, Sulyok M, Warth B, Beltrán E, Krska R, Obadina O, Bakare A and Chilaka CA. (2014). Fungal and bacterial metabolites of stored maize (Zea mays L.) from five agro-ecological zones of Nigeria. Mycotoxin Res. 30(2):89-102.

Ahuja M, Bishnoi M and Chopra KT. (2008). Protective effect of minocycline, a semi-synthetic second-generation tetracycline against 3-nitropropionic acid (3-NP)-induced neurotoxicity. 
International Nuclear Information System (INIS). Toxicol, 244: (2-3): 111-122.

Alonso VA, Pereyra CM, Keller LAM, Dalcero AM, Rosa CAR and Chiacchiera SM. (2013). Fungi and mycotoxins in silage: An overview. J of Appl Microbiol, 115:(3):637- 643.

Alsohaili SA and Bani-Hasan BM. (2017). Morphological and Molecular Identification of Fungi Isolated from Different Environmental Sources in the Northern Eastern Desert of Jordan. Jordan J Biol Sci., 11(3):229-337.

Amber P, Akram A, Qureshi R and Arid S,. (2012). HPLC analysis for secondary metabolites detection in Sclerotium rolfsii isolated from chickpea. Pak. J. Bot. 44: 417-422.

Audebert M, Theumeur M, Henneb Y, Payros D, Borin C, Tadrist S, Beltz S, Puel O and Oswald I. (2014). Genotoxicity of aflatoxin precursors. Abstracts of Lectures and Posters of theWorld Mycotoxin Forum $8^{\text {th }}$ Conference, Wien, Austria, 10-12 November 2014, 43: 102.

Bakhiet SEA and Musa AAA. (2011). Survey and Determination of Aflatoxin Levels in Stored Peanut in Sudan: J Biol Sci., 4(1): 13-20.

Berthiller F, Brera C, Crews C, Iha MH, Krska R, Lattanzio VMT, MacDonald S, Malone R. J, Maragos C, Solfrizzo M, Stroka J and Whitaker TB. (2015). Developments in mycotoxins analysis: an update for 2013-2014. World Mycotoxin J., 8: 5-35.

Berthiller F, Crews C, Dall'Asta C, De Saeger S, Haesaert G, Karlovsky P, Oswald IP, Seefelder W, Speijers G and Stroka J. (2013). Masked mycotoxins: a review. Mol. Nutr. Fd Res. 57: 165-186.

Bertuzzi T, Romani M, Rastelli S, Mulazzi A, and Pietri A. (2017). Sterigmatocystin Occurrence in Paddy and Processed Rice Produced in Italy in the Years 2014-2015 and Distribution in Milled Rice Fractions. Toxins 9 (86) : 1-11.

Bhatnagar-Mathur P, Sunkara S, Bhatnagar-Panwar M, Waliyar F and Sharma KK. (2015). Biotechnological advances for combating Aspergillus flavus and aflatoxin contamination in crops. Plant Sci. 234: 119-132.

Burdock GA, Soni MG and Carabin IG. (2001). Evaluation of health aspects of kojic acid in food. Regulatory Toxicol and Pharmacol, 33 (1) : 80-101.

Cary, JW. Gilbert, MK, Lebar RM, and Calvo AM: (2018). Aspergillus flavus Secondary Metabolites: More than Just Aflatoxins. Food Saf (Tokyo). 6(1): 7-32. doi: 10.14252/foodsafetyfscj.2017024.

Chang PK, Horn BW and Dorner JW. (2005). Sequence breakpoints in the aflatoxin biosynthesis gene cluster and flanking regions in non-aflatoxigenic Aspergillus flavus isolates. Fungal Genet. Biol, 42:914-923.

Changwa R, Abia W, Msagati, T, Nyoni, H, Ndleve, K and Njobeh, P. (2018). Multi-mycotoxin Occurrence in Dairy Cattle Feeds from the Gauteng Province of South Africa: A Pilot Study Using UHPLC-QTOF-MS/MS. Toxins 10 (7): 294.

da Silva JL, Aparecido CC, Hansen D, Pereira TAM, D’arcFelicio J and Gonçalez E. (2015). Identification of toxigenic Aspergillus species from diet dairy goat using a polyphasic

approach. Ciência Rural, Santa Maria, 45(8):1466-1471.

Dwivedi SL, Crouch JH, Nigam SN, Ferguson ME and Paterson AH.(2003). Molecular breeding of groundnut for enhanced productivity and food security in the semi-arid tropics: opportunities and challenges. Adv in Agron, 80: 153-22.

Garcia-Cela E, Kiaitsi E, Sulyok,M, Krska, R, Medina A and I. Petit Damico IP. (2019). Influence of storage environment on maize grain: $\mathrm{CO}_{2}$ production, dry matter losses and aflatoxins contamination. Food Addit Contam: Part A, 36: 175-185.
Gao W, Jiang L. Ge L, Chen M, Geng C, Yang G, Li, Q, Ji F, Yan Q, Zou Y. (2015). Sterigmatocystin-induced oxidative DNA damage in human liver-derived cell line through lysosomal damage. Toxicol. 29: 1-7.

Giorni P, Bertuzzi T and Battilani P. (2019) Impact of Fungi Cooccurrence on Mycotoxin Contamination in Maize During the Growing Season. Front. Microbiol. 10:1265. doi: 10.3389/fmicb.2019.01265.

Granados-Chinchilla F. (2017). A Focus on Aflatoxin in Feedstuffs: New Developments in Analysis and Detection, Feed Composition Affecting Toxin Contamination, and Interdisciplinary Approaches to Mitigate It, Aflatoxin - Control, Analysis, Detection and Health Risks, Lukman Bola Abdulra'uf, IntechOpen. 12:251-280.

Gruber-Dorninger C, Novak B, Nagl V, and Berthiller F. (2017). Emerging Mycotoxins: Beyond Traditionally Determined Food Contaminants. J of Agric and Food Chem. 65: 7052-7070.

Guchi E. (2015a). Implication of aflatoxin contamination in agricultural products. American J of Food and Nutri. 3 (1):12-20.

Guchi E. (2015b). Aflatoxin Contamination in Groundnut (Arachis hypogaea L.) Caused by Aspergillus Species in Ethiopia." J Appl \& Environ Microbiol. 3(1): 11-19.

Ifeji EI, Makun HA, Mohammed, HL, Adeyemi, RYH, Mailafiya SC, Mohammad KH. and Olurunmowaju YB. (2014). Natural occurrence of aflatoxin and roasted groundnut from Niger State, Nigeria. Mycotoxicology, 1(1): 35-45.

International Organization for Standardization (2015). ISO guide 13528:2015(en) statistical methods for use in proficiency testing by interlaboratory comparison.

Jallow EAA. (2015). Determination of aflatoxin-producing fungi strains and levels of aflatoxin $\mathrm{B}_{1}$ in some selected local grains. MPhil Degree Thesis in Biochemistry. Department of Biochemistry and Biotechnology, Kwame Nkrumah University of Science and Technology (KNUST), Kumasi, Ghana.

Joanne MW, Linda MS and Christopher JW. (2008). Prescott, Harley \&Kleins Microbiology. $7^{\text {th }}$ edition. Mcgraw hill publishers, New York. 1028-1048.

Malachova A, Sulyok M, Beltrán E, Berthiller F, Krska R. (2015). Multi-toxin determination in food - The power of 'Dilute-and-shoot approaches in LC-MS-MS. LCGC Europe, 28(10): 542-555.

Malachová A, Stránská M, Václavíková M. Elliot CT, Black C, Meleely J, Hajslova J, Ezekiel CN, Schuhmacher R and Kriska R. (2018). Advanced LC-MS-based methods to study the cooccurrence and metabolization of multiple mycotoxins in cereals and cereal-based food. Anal Bioanal Chem 410: 801-825.

Nesic K, Ivanovic S and Nesic V. (2014) Fusarial Toxins: Secondary Metabolites of Fusarium Fungi. In: Whitacre D. (eds) Rev Environ Contam Toxicol 228: 101-120.

Nielsen KF, Mogensen JM, Johansen, M Larsen, TO and Frisvad, JC (2009). Review of secondary metabolites and mycotoxins from the Aspergillus niger group. Anal. bioanal Chem. 395:1225-1242.

Ntare BR. (2007). Arachis hypogaea L. In: van der Vossen, H.A.M. \& Mkamilo, G.S. (Editors). PROTA (Plant Resources of Tropical Africa/Ressources végétales de l'Afrique tropicale), Wageningen, Netherlands. Accessed 2 June 2018.

Ranjbar S, Nazari R and Noori M. (2011). Isolation and molecular identification of Aspergillus species of Cattle. Vet. Res. 7(1):1117.

Rank C, Nielsen KF, Larsen TO, Varga J, Samson RA and Frisvad JC. (2011). Distribution of sterigmatocystin in filamentous fungi. Fungal Biol. 115: 406-420. 
Rychlik M, Humpf HU, Marko D, Dänicke S, Mally A, Berthiller F, Klaffke H and Lorenz N. (2014). Proposal of a comprehensive definition of modified and other forms of mycotoxins including “masked” mycotoxins. Mycotoxin Res. 30: 197- 205.

Sulyok M, Krska R and Schuhmacher R. (2010). Application of an LC-MS/MS based multi-mycotoxin method for the semiquantitative determination of mycotoxins occurring in different types of food infected by moulds. Food Chem. 119:408-416.

Vabi MB, Ogara I; Anjorin TS; , Oluwabaniwo F; , Alabi O, Ajeigbe HA and Denloye S. (2018). Aflatoxins in Nigerian groundnut: Continuous threat to health,, agriculture and foreign trade. Policy brief 35, (April, 2018).
Varga E, Wiesenberger G, Hametner C, Ward TJ, Dong Y, Schöfbeck D, McCormick S, Broz K, Stückler R, Schuhmacher R, Krska R, Kistler HC, Berthiller F and Adam G. (2015). New tricks of an old enemy: isolates of Fusarium graminearum produce a type A trichothecene mycotoxin Environ. Microbiol., 17: $2588-2600$.

Versilovskis A and De Saeger SA. (2010). Strigmatocystion: occurrence in foodstuffs and analytical methods: An overview. Mol Nutri and Food Res. 54(1) : 136-147.

Wartu JR, Whong, CMZ, Abdullahi IO, and Ameh JB. (2017). Phylogenetics of aflatoxin from in-process wheat and flour from selected major stores within Northern Nigeria. Sci. World J.12(4): $1-5$ 